\title{
Prediction of the Response of the Commercial BPW34FS Silicon p-i-n Diode Used as Radiation Monitoring Sensors up to Very High Fluences
}

\author{
J. Mekki, M. Moll, M. Fahrer, M. Glaser, and L. Dusseau, Senior Member, IEEE
}

\begin{abstract}
The effect of radiation damage on Silicon p-i-n diodes has been studied. $I-V$ characteristics of BPW34FS silicon p-i-n diodes irradiated with $24 \mathrm{GeV} / \mathrm{c}$ protons up to $6.3 \times 10^{15} \mathrm{n}_{\mathrm{eq}} / \mathrm{cm}^{2}$ have been measured and analyzed. A parameterization predicting the radiation response in the fluence range relevant for the use of the diodes as radiation monitors in Super-LHC experiments is presented.
\end{abstract}

Index Terms-Accelerators, p-i-n diodes, particle beams, radiation damage, radiation monitoring.

\section{INTRODUCTION}

$\mathbf{S}$ ILICON $p-i-n$ diodes are important devices for radiation monitoring in high energy physics experiments in very harsh radiation conditions, such as the Large Hadron Collider (LHC) experiments at CERN [1].

The equivalent fluence $\Phi_{\mathrm{eq}}$ which the detectors are exposed to, covers a wide range from below $10^{8}$ to $10^{14}-10^{15} \mathrm{n}_{\text {eq }} / \mathrm{cm}^{2}$ (1-MeV equivalent neutrons per $\mathrm{cm}^{2}$ ), depending on the location in the LHC experiments.

For the future upgrade of the LHC experiment (Super-LHC) [2], [3], the radiation level will be multiplied by a factor of 10 due to the increase of the luminosity from $10^{34}$ to $10^{35} \mathrm{~cm}^{-2} \mathrm{~s}^{-1}$. For the innermost detectors, the silicon tracking sensors will be exposed to fluences of up to $10^{16} \mathrm{n}_{\mathrm{eq}} / \mathrm{cm}^{2}$. This causes severe radiation damage and calls for a reliable radiation monitoring. In this work the commercial OSRAM BPW34FS silicon p-i-n diode [4] is investigated in view of its application as radiation monitor in Super-LHC experiments. This type of diode was already characterized at CERN in previous studies [5]-[8] in order to use them as radiation monitoring sensors for the LHC experiments.

For monitoring high fluences, p-i-n diodes are used in forward bias mode by applying a constant readout current pulse of short duration to avoid self-heating effects. Using this method, sensitivity to fast hadrons has been observed up to high fluences by measuring the variation of the forward voltage versus $\Phi_{\text {eq }}$.

Manuscript received September 11, 2009; revised January 08, 2010; accepted February 10, 2010. Date of current version August 18, 2010.

J. Mekki is with CERN, CH-1211 Geneva 23, Switzerland, and also with the IES, Montpellier, France (e-mail: Julien.Mekki@ cern.ch).

M. Moll, M. Fahrer, and M. Glaser are with CERN, CH-1211 Geneva 23, Switzerland.

L. Dusseau is with the IES-UMR UMII-CNRS 5214, University Montpellier II, F-34095, Montpellier Cedex 5, France.

Digital Object Identifier 10.1109/TNS.2010.2044191
The previous works showed that the commercial p-i-n diode powered at $1 \mathrm{~mA}$ in forward bias operation with a pulse duration of $700 \mathrm{~ms}$ was a valuable choice, since diodes revealed a hadron sensitivity range between $2 \times 10^{12} \mathrm{n}_{\mathrm{eq}} / \mathrm{cm}^{2}$ and $4 \times 10^{14} \mathrm{n}_{\mathrm{eq}} / \mathrm{cm}^{2}$, which was sufficient for monitoring hadron fluences as expected for the LHC.

However, for monitoring even higher radiation levels as needed for the future Super-LHC experiments, a solution for expanding the fluence measurement range has to be found.

In this work we present a detailed experimental investigation into the response of BPW34FS silicon p-i-n diodes exposed to $24 \mathrm{GeV} / \mathrm{c}$ proton fluences of up to $6.3 \times 10^{15} \mathrm{n}_{\mathrm{eq}} / \mathrm{cm}^{2}$.

In order to parameterize the obtained data, a theoretical approach based on the relaxation material semiconductor theory as described in [9]-[16] has been used in this work. It should be clearly pointed out that this approach was used because the mathematical formulation could conveniently and successfully be used to fit and parameterize the obtained experimental data. Other theoretical approaches towards the description of $I-V$ curves, like for example used in [17]-[19] for diodes irradiated with neutrons up to $1 \times 10^{13} \mathrm{n}_{\mathrm{eq}} / \mathrm{cm}^{2}$, follow the classical semiconductor theory and might thus be regarded as more fundamental and sound. However, aim of this work is not to compare these theoretical approaches, but to parameterize the obtained data. The relaxation material approach, as described in detail in [9]-[16] and briefly summarized in Section IV, delivered a good basis for the parameterization and was therefore used in this work.

The diode forward voltage response as function of particle fluence and injection current was parameterized in a wide range of validity and allowed to extend the measurement range of the diodes up to about $6.3 \times 10^{15} \mathrm{n}_{\mathrm{eq}} / \mathrm{cm}^{2}$. Furthermore, the parameterization now allows to predict the forward voltage response for any injection current $I_{F} \leq 1 \mathrm{~mA}$. The latter feature allows optimizing the injection current for the fluence range of interest. Finally, a preliminary study on the temperature dependence of the diode response for different injection currents is presented. It shall be clearly stated that the results in this work are entirely based on the commercial BPW34FS diodes. Custom made diodes would obviously allow for further device modifications in terms of e.g., the diode base length and diode shape (see e.g., [17] and [19]), but remain to be developed, tested and optimized for the high fluences expected for the Super-LHC application.

This paper is organized in eight sections. In Section II we describe the diode and the measurement method. A discussion 
on the measured forward and reverse $I-V$ characteristics of the BPW34FS diodes as function of proton fluence is given in Section III. Section IV summarizes the relaxation theory and gives the basis for the later data parameterization. In Section $\mathrm{V}$, the $I-V$ curves are analyzed in terms of the relaxation theory approach and finally the data are fitted and parameterized up to very high fluences in Section VI.

In Section VII, the temperature dependence of irradiated diode has been qualitatively evaluated with the intention to give some suggestions about the optimal temperature in which the diode should be used for monitoring radiation as expected in the future Super-LHC. Section VIII gives the conclusions.

\section{MEASUREMENT}

A detailed description of the used device can be found in [7] and [8]. While for these previous studies, the diodes were packaged with the configuration of type BPW34F (hosted in a plastic packaging), another packaging configuration was employed for the work presented here. The diode is encapsulated in a SMD plastic packaging, which allows easy integration on a PCB board.

The commercial BPW34FS silicon p-i-n diode active area is $2.65 \times 2.65 \mathrm{~mm}^{2}$ with a base thickness of about $300 \mu \mathrm{m}$ [8].

The BPW34FS silicon p-i-n diodes were exposed to the 24 $\mathrm{GeV} / \mathrm{c}$ proton beam of the IRRAD1 facility at CERN [20] with fluences ranging from $1.4 \times 10^{11}$ up to $6.3 \times 10^{15} \mathrm{n}_{\mathrm{eq}} / \mathrm{cm}^{2}$. Irradiations were carried out at about $27^{\circ} \mathrm{C}$ with a fluence accuracy within $\pm 7 \%$.

The current-voltage characteristic measurements were carried out at room temperature before and after irradiations. For any given fluence value an individual diode was irradiated. In order to evaluate the accuracy of the measurement setup, diodes were contacted and measured several times at room temperature showing no measurable difference. The sample to sample variation after irradiation was not studied in this work as only one sample was irradiated per fluence. However, accuracy is known to be better than $10 \%$ according to a previous study which used another measurement setup with a higher systematic uncertainty [21].

The samples were irradiated at about $27^{\circ} \mathrm{C}$ and have therefore undergone some annealing during irradiation. According to previous measurements performed with $I_{F}=1 \mathrm{~mA}$ ([7] and [8]) the corresponding drop in signal should be less than about $3 \%$ up to a fluence of $1 \times 10^{14} \mathrm{~cm}^{-2}$. For higher fluences (and the corresponding longer exposure times) no annealing data exist so far. In order to avoid room temperature annealing after irradiation, the diodes were stored in a deep freezer below $-20^{\circ} \mathrm{C}$ when not measured.

Since the BPW34FS silicon p-i-n diode is light sensitive, measurements have been performed in a light-tight box, using a test bench based on a Keithley 2400 (or 2410 for voltages higher than 210 Volts) and a Keithley 485 picoamperemeter. From here on, Keithley 2400 and 2410 will be named Keithley 24XX, for a better readability. The temperature during measurements was recorded by placing a PT100 temperature sensor close to the diode. The average temperature during measurement was of $20.7 \pm 0.2^{\circ} \mathrm{C}$, corresponding to the room temperature.
Reverse bias measurements were done using the Keithley $24 \mathrm{XX}$ as d.c voltage source, from $-1 \mathrm{mV}$ to $-500 \mathrm{~V}$. The reverse current was measured by the picoamperemeter and limited to a compliance of $1 \mathrm{~mA}$ in order to avoid possible self-heating effects at high current.

In addition, forward measurements were carried out in the following steps:

1) For the lower current values $\left(I_{F} \leq 100 \mathrm{nA}\right)$, the measurements were performed as for reverse bias, i.e by sourcing voltages with the Keithley $24 \mathrm{XX}$ and by measuring the forward currents with the picoamperemeter. This method was selected since for measurement of low current values, the Keithley 485 is more precise than the Keithley 24XX.

2) From $100 \mathrm{nA}$ to $1 \mathrm{~mA}$, measurements were done only with the Keithley 24XX, by injecting short current pulses of 50 ms pulse duration and by measuring the corresponding forward voltages. In order to avoid self heating effects inside the detector, it is better to reduce the measurement time by taking only few points at high readout current. Thus, at $I_{F}>1 \mathrm{~mA}$, measurements were carried out by injecting only 8 current steps: $2 \mathrm{~mA}, 3 \mathrm{~mA}, 4 \mathrm{~mA}, 5 \mathrm{~mA}, 10 \mathrm{~mA}$, $15 \mathrm{~mA}, 20 \mathrm{~mA}$ and $25 \mathrm{~mA}$.

\section{EXPERIMENTAL OBSERVATIONS}

Forward and reverse $I-V$ characteristics of unirradiated and irradiated BPW34FS silicon p-i-n diodes have been performed. Measurements were obtained after exposures with various proton fluences ranging from $1.4 \times 10^{11}$ to $6.3 \times 10^{15} \mathrm{n}_{\mathrm{eq}} / \mathrm{cm}^{2}$. The proton fluence was converted into $1-\mathrm{MeV}$ neutron equivalent fluence $\left(\Phi_{\text {eq }}\right)$ by the experimentally obtained hardness factor $(\mathrm{k})$ of 0.62 [22]. Results are presented in log-log scale in Figs. 1 and 2 (forward $I-V$ characteristics only). For a better visibility only a few measurements are shown.

Two different regions can be distinguished in the $I-V$ characteristics.

For the non-irradiated diode, at low voltages up to around 20 $\mathrm{mV}$ in both operation directions, a linear region is observed. As the voltage increases, the curve shape changes. While for forward bias measurements there is a change from the linear region to a sharp increase of the forward current, for reverse bias measurements only a slight increase of the reverse current appears. At even higher reverse voltages, the current starts to increase rapidly indicating that the device is approaching breakdown.

For reverse bias operation mode, as the radiation level increases, a rise of the current versus fluence can be observed. For forward bias, at voltages lower than around $300 \mathrm{mV}$, an increase of the current versus fluence can also be observed.

In both operation modes and for fluences above $1 \times 10^{13} \mathrm{n}_{\mathrm{eq}} / \mathrm{cm}^{2}$, there is a linear region at low voltages $(V<300 \mathrm{mV})$. Because the current increases versus radiation damage, this linear region appears at higher currents than the one observed for unirradiated diodes. With further increase of the radiation level, this linear behavior extends to even higher voltages.

In order to obtain a formulation to parameterize the diode's radiation response, a comparison between irradiated silicon 


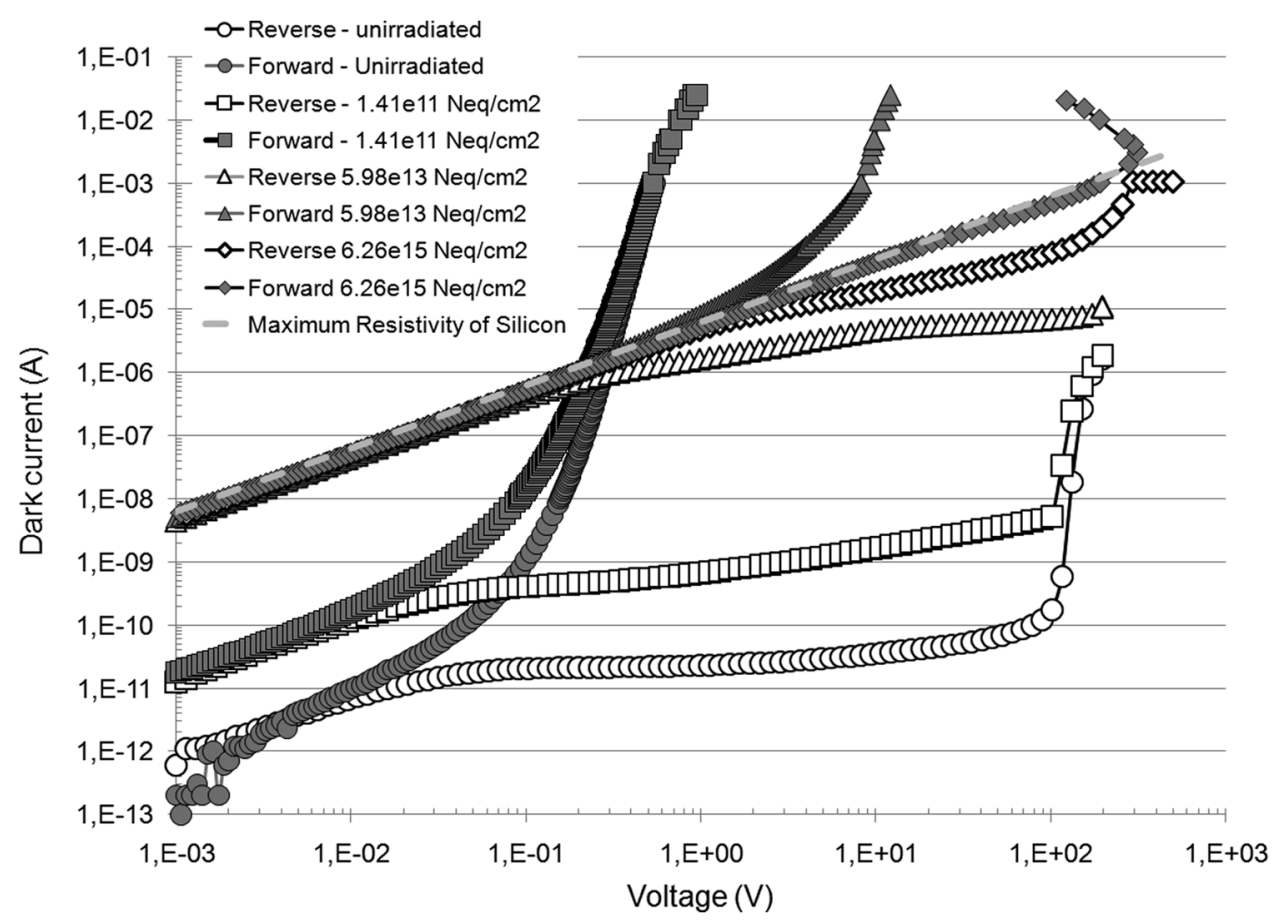

Fig. 1. I-V characteristics for forward and reverse bias of non-irradiated and irradiated p-i-n diodes after $24 \mathrm{GeV} / \mathrm{c}$ proton irradiation. Measurements were performed at room temperature. The dashed line represents the maximum resistance at $300 \mathrm{~K}$. $I_{R}$ values $>1 \mathrm{~mA}$ have not been plotted, since the reverse current was limited to a compliance of $1 \mathrm{~mA}$.

p-i-n diodes and relaxation materials will be given in Section IV following the discussions given in [9]-[16].

\section{Summary of the Relaxation Regime AND DIODE PROPERTIES}

The lattice destruction by protons or neutrons introduces deep level defects which modify the electrical properties of the silicon material. These defects increase the resistivity of the semiconductor material and are responsible for its semi-insulating behavior, which has been attributed to the relaxation-like properties in semiconductors as it is referenced in [23]-[25].

According to the relaxation material theory, a material becomes relaxation-like, if it has a high resistivity and a large density of generation-recombination (g-r) centers. Such traps can be produced by radiation damage in silicon $\mathrm{p}-\mathrm{i}-\mathrm{n}$ diodes.

The dielectric relaxation time $\tau_{D}[26]$ is the time for restoring neutrality to a region when excess carrier are introduced and is expressed by the following equation:

$$
\tau_{D}=\rho \varepsilon \varepsilon_{0}
$$

where $\rho$ is the material resistivity, $\varepsilon_{0}$ is the relative dielectric constant of silicon and $\varepsilon_{0}$ is the permittivity of free space.

In relaxation material, the resistivity increases since the concentration of both minority and majority carriers is reduced by recombination at the g-r centers. As radiation damage increases, the dielectric relaxation time becomes higher than the minority carrier lifetime $\tau_{0}$ such as $\tau_{D} \gg \tau_{0}$.

In addition, as material becomes relaxation-like if it has a large density of defect g-r electronic levels near the middle of the energy gap $\left(E_{g}\right)$ which can readily interact with both types of carriers, recombination pins the Fermi levels at the value of minimum conductivity [23]. Therefore, the material reaches the maximum resistivity $\rho_{\max }$ which is given by the following expression:

$$
\rho_{\max }=\frac{1}{2 q\left(\mu_{n} \mu_{p}\right)^{1 / 2} n_{i}}
$$

In (2), $\mathrm{q}$ is the elementary charge, $\mu_{n}=1450 \mathrm{~cm}^{2} \cdot \mathrm{V}^{-1} \cdot \mathrm{s}^{-1}$ and $\mu_{p}=502 \mathrm{~cm}^{2} \cdot \mathrm{V}^{-1} \cdot \mathrm{s}^{-1}$ are the electron and hole mobilities respectively and $n_{i}$ is the intrinsic carrier concentration which is equal to $9.65 \times 10^{9} \mathrm{~cm}^{-3}$, all at $300 \mathrm{~K}$ [27].

Then $\rho_{\max }$ is around $3.8 \times 10^{5} \Omega \cdot \mathrm{cm}$ for silicon.

Reverse and forward measurements are presented in Fig. 1 with a dashed line representing the maximum resistance of the $\mathrm{p}$ - $\mathrm{i}-\mathrm{n}$ diodes. This value is derived from the relation

$$
I=\frac{A}{\rho_{\max } L} V
$$

where $A$ and $L$ are the active area and thickness of the diode respectively and $\rho_{\max }$ is the maximum resistivity of silicon.

\section{ANALYSIS OF $I-V$ CURVES}

First, reverse measurements are discussed. As it is expected from the conventional lifetime theory [27], for unirradiated diodes, at voltage superior to $3 \mathrm{kT} / \mathrm{q}(78 \mathrm{mV})$ only a slight increase of the current versus voltage is observed arising from small concentrations of g-r centers present in the unirradiated silicon and surface leakage of the diode. At higher reverse voltages, the current rises sharply which indicates the occurrence of the avalanche breakdown. 


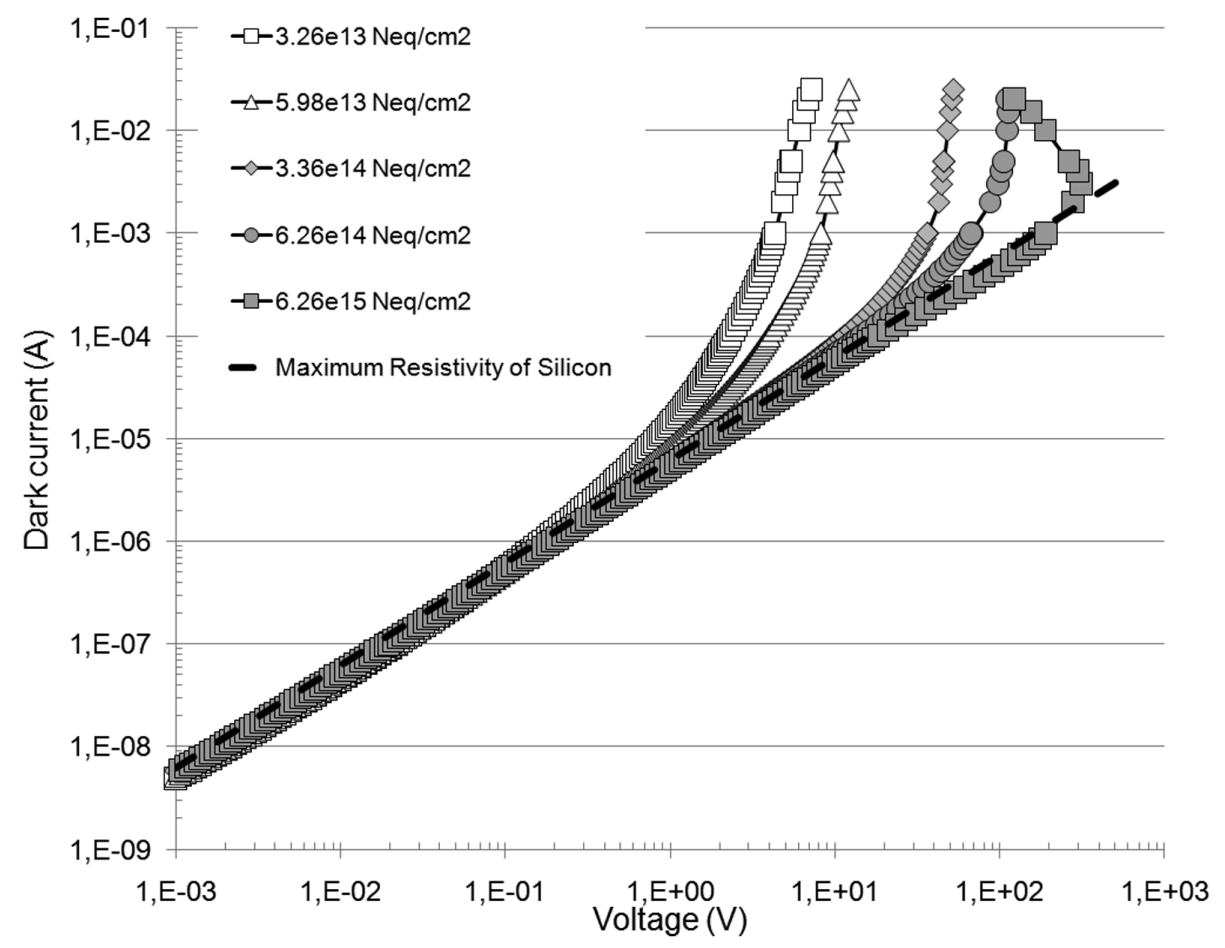

Fig. 2. I- $V$ characteristics for forward bias of irradiated p-i-n diodes after $24 \mathrm{GeV} / \mathrm{c}$ proton irradiation and $\Phi_{\mathrm{eq}}>10^{13} \mathrm{n}_{\text {eq }} / \mathrm{cm}^{2}$ with the corresponding maximum resistance line at $300 \mathrm{~K}$. Results presented here are taken from Fig. 1, but for a better visibility only forward measurements are illustrated and some radiation levels are added for completeness.

For irradiated diodes, as radiation damage increases with fluence, more g-r centers are created in the silicon bulk leading to an increase of reverse current. For low voltages (in the linear region, where $V_{R}<78 \mathrm{mV}$ ) the reverse current versus $\Phi_{\text {eq }}$ increases until it reaches a maximum value for $\Phi_{\mathrm{eq}}>1 \times$ $10^{13} \mathrm{n}_{\mathrm{eq}} / \mathrm{cm}^{2}$. For $\Phi_{\mathrm{eq}}>1 \times 10^{13} \mathrm{n}_{\mathrm{eq}} / \mathrm{cm}^{2}$, this linear region extends to higher voltages as the radiation level increases. For higher voltages (not in the linear region), it can be mentioned that the generation current dominates, as in reverse bias, traps alternately emit electrons and holes. In this case, the generation current dominates and gives the $V^{1 / 2}$ variation corresponding to the increase of the depletion width [27].

In the forward bias regime, for $\Phi_{\mathrm{eq}}<10^{13} \mathrm{n}_{\mathrm{eq}} / \mathrm{cm}^{2}$, the $\mathrm{p}$-i-n diode behaves as conventional lifetime diode, with high current flowing in the forward direction due to the diffusion current. The conventional lifetime diode has $\tau_{D} \ll \tau_{0}$, charge neutrality is always assumed in the depletion region, and then minority carrier injection reduces the resistivity since the total concentration of carriers is enhanced by the increase in both majority and minority carrier. In lifetime diode, dielectric relaxation occurs quickly and charge flows rapidly to cancel any non-equilibrium space charge and local charge neutrality is a basic equilibrium requirement [15], [16] (contrary to the relaxation regime, where $\tau_{D} \gg \tau_{0}$ ).

At higher currents, the p-i-n diode may enter in high injection state i.e the injected minority carrier density becomes comparable to the doping density in the n-substrate [27].

In forward bias, at low voltages, in the linear region (where $V_{F}<78 \mathrm{mV}$ ), with increasing fluences, a growth of the current can be observed, up to reach a maximum value for $\Phi_{\text {eq }}>1 \times$
$10^{13} \mathrm{n}_{\mathrm{eq}} / \mathrm{cm}^{2}$. This saturation occurs at the same current value than the one observed for the reverse bias mode.

For both operation modes, at $\Phi_{\mathrm{eq}}>1 \times 10^{13} \mathrm{n}_{\mathrm{eq}} / \mathrm{cm}^{2}$, the ohmic-like behavior appears and the $I-V$ characteristics are overlapping to the $I-V$ ohmic characteristic corresponding to maximum resistivity of compensated base of the diode, as illustrated in Figs. 1 and 2, which indicates that the material is becoming ohmic-like when radiation damage increases.

\section{CHARACTERIZATION OF THE FoRWARD BIAS RESPONSE}

In previous studies [13], [15] and [16] it has been found that in forward bias the $I-V$ characteristic of irradiated silicon p-i-n diodes fits the relation

$$
I=G_{0} V \exp \left(\frac{V}{V_{0}}\right)
$$

where $G_{0}$ is the conductance in unit of $\Omega^{-1}$ defined by the following formula:

$$
G_{0}=\left(\frac{1}{R_{0}}\right)=\left(\frac{A}{\rho \times L}\right)
$$

In this study, the value of $G_{0}$ has been evaluated from $I-V$ curves of diodes irradiated at $\Phi_{\mathrm{eq}}>1 \times 10^{13} \mathrm{n}_{\mathrm{eq}} / \mathrm{cm}^{2}$ as $G_{0}=$ $\left(4.90 \times 10^{-6} \pm 6.43 \times 10^{-8}\right) \Omega^{-1}$. $A$ and $L$ are the diode's active area and thickness which are equal to $70.225 \times 10^{-3} \mathrm{~cm}^{2}$ and $3 \times 10^{-2} \mathrm{~cm}$, respectively.

Equation (4) was used earlier as an empirical fitting function for relaxation materials [28]. Applying such parameterization implies some assumptions, as that the details of contacts; the 


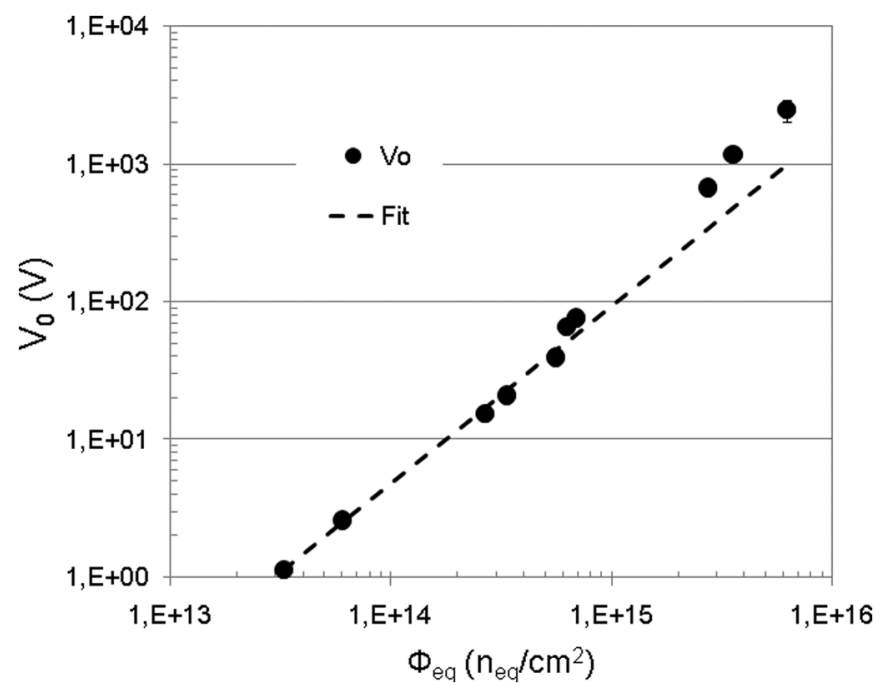

Fig. 3. The value of the fitting parameter $V_{0}$ as a function of equivalent fluences.

necessary conditions are that the material has high resistivity, that it is a nearly intrinsic or compensated semi-conductor and that it has a very high density or g-r centers [16]. This empirical fitting expression successfully allows to parameterized the $I-V$ curves of the type of diode considered in this work, for $\Phi_{\text {eq }}>$ $1 \times 10^{13} \mathrm{n}_{\mathrm{eq}} / \mathrm{cm}^{2}$ and $I_{F} \leq 1 \mathrm{~mA}$ (this value is discussed later).

As it can be observed here, the fitting parameter $G_{0}$ is not fluence dependent, contrary to [29], where the $G_{0}$ values decreases versus fluences between $3.4 \times 10^{13} \mathrm{n}_{\mathrm{eq}} / \mathrm{cm}^{2} \leq \Phi_{\mathrm{eq}} \leq$ $2.5 \times 10^{14} \mathrm{n}_{\mathrm{eq}} / \mathrm{cm}^{2}$.

$G_{0}$ depends on the maximum resistivity of silicon $(\rho)$. Since the latter one depends on carrier mobility and intrinsic carrier concentration as it is shown in (2), if the temperature during measurement is stable enough, the value of $\rho_{\max }$ should be constant as the carrier mobilities in irradiated silicon do not vary versus fluences up to $2.4 \times 10^{14} \mathrm{n}_{\mathrm{eq}} / \mathrm{cm}^{2}$ [29].

Contrary to $G_{0}$, the fitting parameter $V_{0}$ is dependent on the radiation level as illustrated in Fig. 3. The dependence of $V_{0}$ according to the equivalent fluence can be described by an empiric expression of the following form:

$$
V_{0}=\alpha \phi_{\mathrm{eq}}^{\beta}
$$

Here, the parameters $\alpha$ and $\beta$ were found to be equal to $(4.27 \pm 0.37) \times 10^{-18} \mathrm{~V} \cdot \mathrm{cm}^{2}$ and $(1.289 \pm 0.003)$, respectively.

Forward $I-V$ characteristics and fitting curves for $6.26 \times 10^{14} \mathrm{n}_{\mathrm{eq}} / \mathrm{cm}^{2}$ and $3.57 \times 10^{15} \mathrm{n}_{\mathrm{eq}} / \mathrm{cm}^{2}$ are presented in Fig. 4.

As it is shown in this figure, the $I-V$ curves based on (4) fit properly the experimental measurements up to about $1 \mathrm{~mA}$. At higher readout current experimental values deviate strongly from the fitting curves. With increasing readout current the $I-V$ curves start to bend back towards smaller $V_{F}$. This phenomenon is assumed to be caused by two effects. First, at such high fluences the p-i-n diode can exhibit a thyristor-like behavior (see [7] and [8]) and, second, due to the high current the temperature

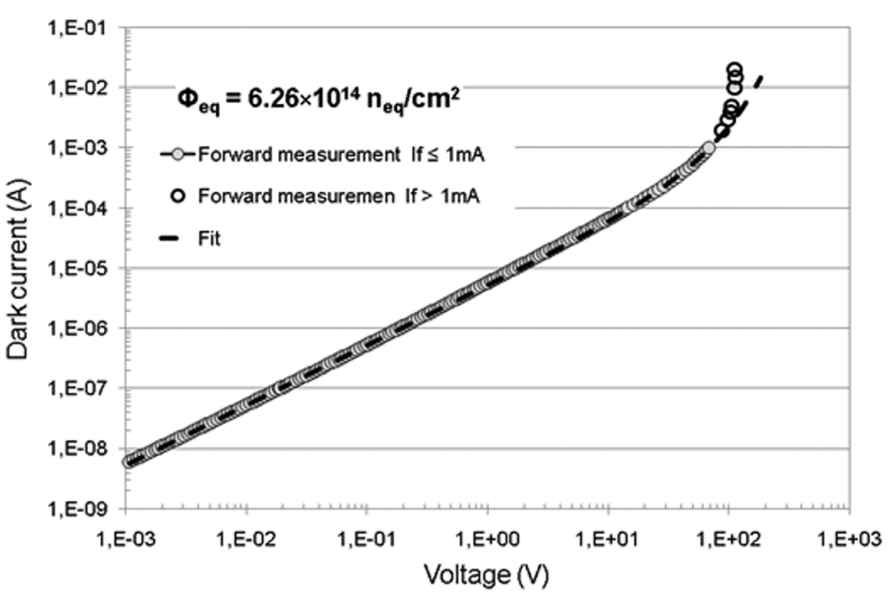

(a)

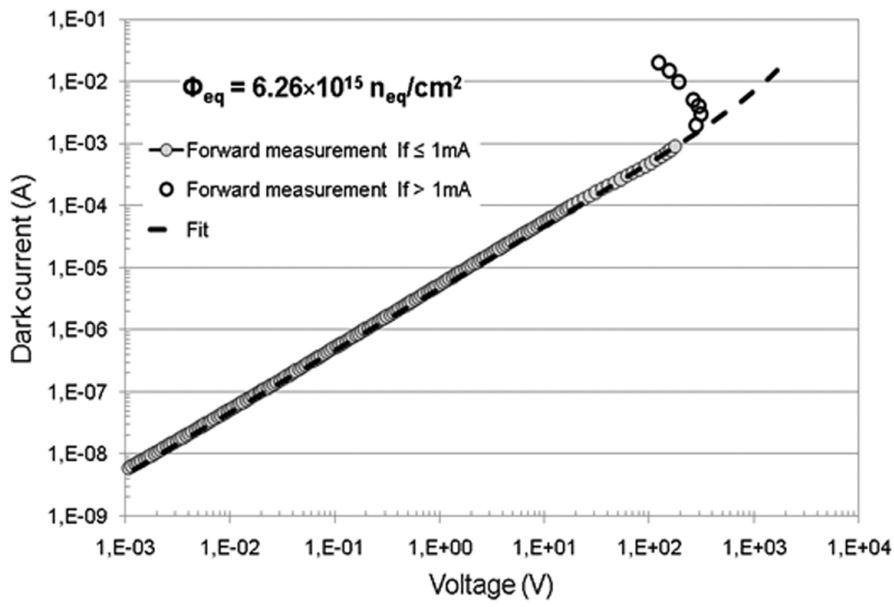

(b)

Fig. 4. Experimental forward characteristics and fitting curves (a) at $\Phi_{\mathrm{eq}}=$ $6.26 \times 10^{14} \mathrm{n}_{\text {eq }} / \mathrm{cm}^{2}$ and (b) at $\Phi_{\text {eq }}=6.26 \times 10^{15} \mathrm{n}_{\text {eq }} / \mathrm{cm}^{2}$ of BPW34FS diodes. Measurements were carried out at room temperature. Empty circles represent measurements at high injection levels where a deviation from the fitting curve can be observed.

increases inside the p-i-n diode. Since silicon has a negative temperature coefficient, if the device is self heated by high current injection, the voltage tends to lower values (see [21]).

Taking into account (4), when $V$ is much lower than $V_{0}$ the diode has an ohmic-like behavior and (4) reduces to

$$
I=G_{0} \times V=\frac{V}{R_{0}}
$$

where $R_{0}$ is the maximum diode's resistance, when the relaxation regime is established. As the voltages increases and becomes higher than $V_{0}$, the current growths exponentially.

For $\Phi_{\text {eq }}>1 \times 10^{15} \mathrm{n}_{\mathrm{eq}} / \mathrm{cm}^{2}$ and high injection currents, voltages bend back to lower values and $I-V$ curves are not governed anymore by the relaxation material equation.

As a consequence, the value $V_{0}$ extracted from the fitting curve of $I-V$ characteristics (e.g., in Fig. 4(b)) does not fit the data for very high fluences (see Fig. 3). Since this phenomenon appears at $I_{F}>1 \mathrm{~mA}$ for the highest radiation levels, the validity of the above given parameterization has to be limited to $I_{F} \leq 1 \mathrm{~mA}$. 


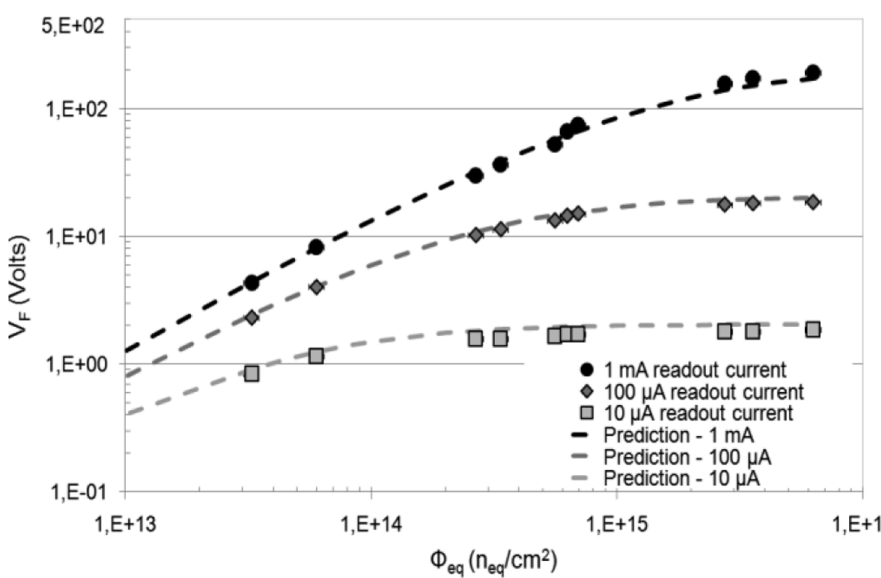

Fig. 5. Experimental and predicted radiation responses of BPW34FS p-i-n diodes at room temperature for equivalent fluences higher than $10^{13} \mathrm{n}_{\mathrm{eq}} / \mathrm{cm}^{2}$. The forward voltage was measured using different readout currents of $50 \mathrm{~ms}$ duration.

For $I_{F}>1 \mathrm{~mA}$, the diode can be regarded to be in a "thyristor regime". The current value of $1 \mathrm{~mA}$ is therefore considered to be the optimum maximum readout current value for BPW34FS diodes when monitoring fluences up to $6.3 \times 10^{15} \mathrm{n}_{\text {eq }} / \mathrm{cm}^{2}$. As pointed out in [7] and [8], $I_{F}=25 \mathrm{~mA}$ can be defined as upper limit if only fluences below $4 \times 10^{14} \mathrm{n}_{\mathrm{eq}} / \mathrm{cm}^{2}$ shall be monitored.

In the framework of the RadMON project [30], [31] at CERN, BPW34FS silicon PIN diodes are used as radiation monitoring sensors to follow on-line the evolution of the LHC fluence. For this reason, BPW34FS diodes are characterized and operated in forward bias.

In this operation mode, it is more reliable to inject current and control voltage since in forward polarization, the current increases sharply versus voltage.

It is therefore convenient to have an expression of the forward voltage versus the readout current as shown in (8):

$$
V=V_{0} \times \operatorname{Lambert} W\left(\frac{I}{G_{0} V_{0}}\right) .
$$

The LambertW(x) function [32] is defined as the inverse function of

$$
f(x)=x \times \exp (x) .
$$

Finally, it can be stated that a formulation for predicting the variation of the forward voltage versus $\Phi_{\text {eq }}$ when the diode is operated at constant readout current was obtained as

$$
V_{F}=\alpha \phi_{\mathrm{eq}}^{\beta} \times \text { Lambert } W\left(\frac{I_{F}}{G_{0} \times \alpha \phi_{\mathrm{eq}}^{\beta}}\right) .
$$

Thanks to this formulation, the radiation response of the OSRAM BPW34FS silicon p-i-n diode operated at constant readout current can be precisely predicted up to very high fluences with two parameters $\left(G_{0}\right.$ and $\left.V_{0}\right)$.

The LambertW function has previously been used to obtain the voltage-current dependence in Schottky barrier diode with inclusion of series resistance and shunt conductance and in solar

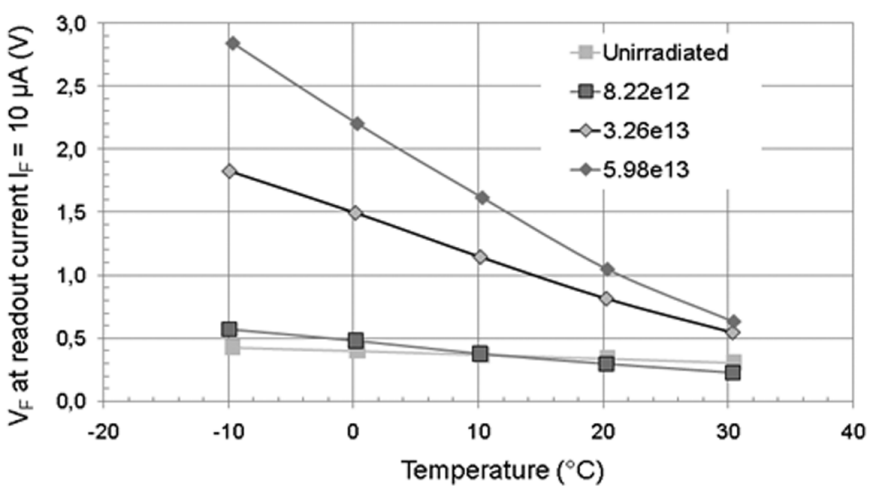

(a)

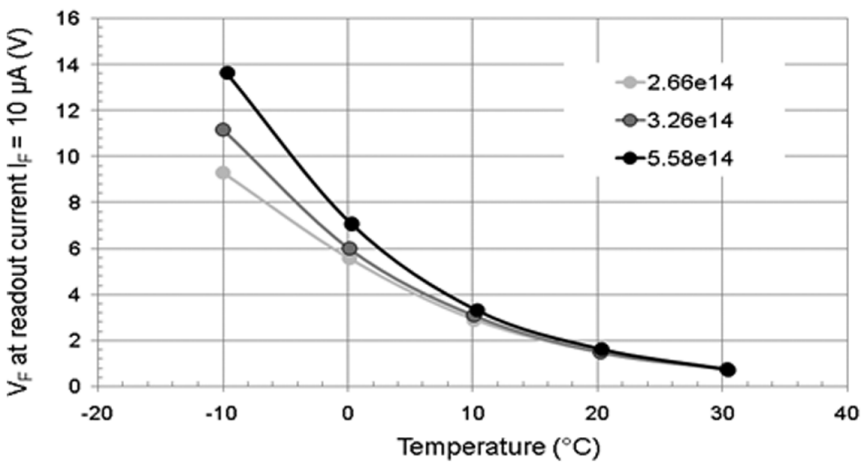

(b)

Fig. 6. Temperature dependence on the BPW34FS diode forward voltage. Measurements have been performed at a readout current $I_{F}=10 \mu \mathrm{A}$ of $50 \mathrm{~ms}$ pulse duration for (a) $\Phi_{\text {eq }} \leq 5.98 \times 10^{13} \mathrm{n}_{\text {eq }} / \mathrm{cm}^{2}$ and for (b) $\Phi_{\text {eq }}>5.98 \times 10^{13} n_{\text {eq }} / \mathrm{cm}^{2}$. Each curve corresponds to a different $\Phi_{\text {eq }}$ as indicated in the legend.

cells [33]-[35]. However, to our best knowledge, in the work presented here, the formulation is used for the first time to predict the variation of the forward voltage versus fluence for irradiated silicon diodes. It allows to predict OSRAM BPW34FS diode's radiation response for $\Phi_{\mathrm{eq}} \geq 10^{13} \mathrm{n}_{\mathrm{eq}} / \mathrm{cm}^{2}$ up to very high fluences and for readout currents $I_{F} \leq 1 \mathrm{~mA}$.

Fig. 5 illustrates experimental and predicted values of the BPW34FS p-i-n diode, for all $\Phi_{\text {eq }} \geq 10^{13} \mathrm{n}_{\mathrm{eq}} / \mathrm{cm}^{2}$ and for readout current $I_{F} \leq 1 \mathrm{~mA}$.

\section{Qualitative Evaluation of the TEMPERATURE DEPENDENCE}

The BPW34FS forward voltage versus equivalent fluence dependence for five different temperatures has been qualitatively evaluated in this study.

The approach to use BPW34FS silicon p-i-n diodes as radiation monitoring sensors consists in operating the device with low readout current since at high readout current the diode enters in thyristor regime.

Moreover, measurements were carried out at low readout current, since it has been shown that in this regime the irradiated BPW34FS p-i-n diode assumes a "relaxation-like" behavior that can be predicted.

The temperature dependence was studied operating the silicon p-i-n diode with $10 \mu \mathrm{A}$ and $100 \mu \mathrm{A}$ readout currents as it is illustrated in Figs. 6 and 7 respectively. Since silicon has a 


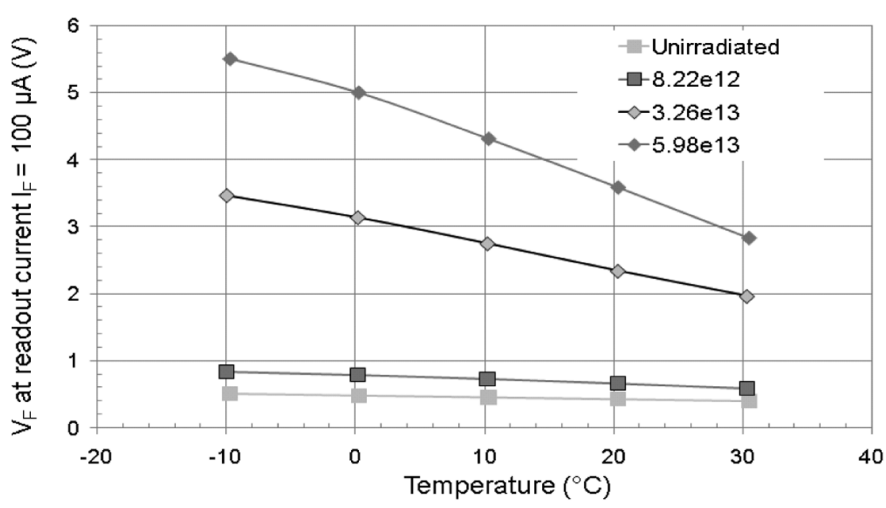

(a)

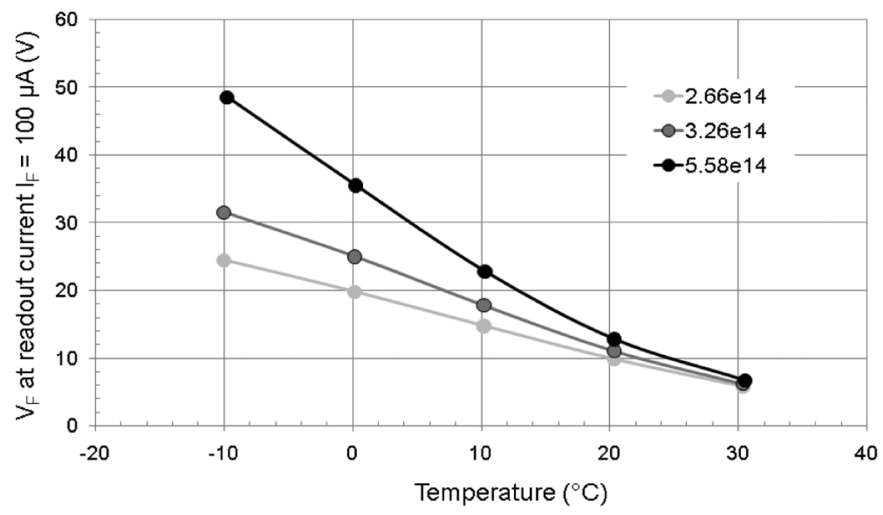

(b)

Fig. 7. Temperature dependence on the BPW34FS diode forward voltage. Measurements have been performed at a readout current $I_{F}=100 \mu \mathrm{A}$ of $50 \mathrm{~ms}$ pulse duration for (a) $\Phi_{\text {eq }} \leq 5.98 \times 10^{13} \mathrm{n}_{\text {eq }} / \mathrm{cm}^{2}$ and for (b) $\Phi_{\text {eq }}>5.98 \times 10^{13} \mathrm{n}_{\text {eq }} / \mathrm{cm}^{2}$. Each curve corresponds to a different $\Phi_{\text {eq }}$ as indicated in the legend.

negative temperature coefficient, the forward voltage decreases as the temperature grows. Furthermore, it can be observed that at high irradiation levels the forward voltage becomes independent on radiation damage, since for different fluences, $V_{F}$ tends to saturate.

For example, as it is shown in Fig. 6(b), measurements performed at $-10^{\circ} \mathrm{C}$ of irradiated diodes $\left(\Phi_{\text {eq }}>\right.$ $\left.1 \times 10^{14} \mathrm{n}_{\mathrm{eq}} / \mathrm{cm}^{2}\right)$ at $I_{F}=10 \mu \mathrm{A}$, reveal that the diode's forward response is fluence dependent. Contrary, for measurement carried out at $30^{\circ} \mathrm{C}, V_{F}$ remains constant.

This effect can be explained since at this temperature, the BPW34FS p-i-n diode operated at $I_{F}=10 \mu \mathrm{A}$, is ohmic-like at already maximum silicon resistivity (see Fig. 8). Therefore, for $\Phi_{\text {eq }}$ higher than around $10^{14} \mathrm{n}_{\mathrm{eq}} / \mathrm{cm}^{2}$, the BPW34FS diode is no more sensitive to increasing radiation damage.

Results of the forward voltage measured at constant readout current versus equivalent fluence for different temperatures are shown in Fig. 8.

As it can be seen in Fig. 8(a), for $\Phi_{\text {eq }}>1 \times 10^{14} \mathrm{n}_{\mathrm{eq}} / \mathrm{cm}^{2}$ the forward voltage versus fluence remains constant or slightly increases. This observation indicates that in the temperature range from $-10^{\circ} \mathrm{C}$ to $30^{\circ} \mathrm{C}$ at such readout current, the diode behaves like a resistance with a maximum resistivity near $\rho_{\max }$. Nevertheless, it can be noticed that as the temperature decreases, $V_{F}$

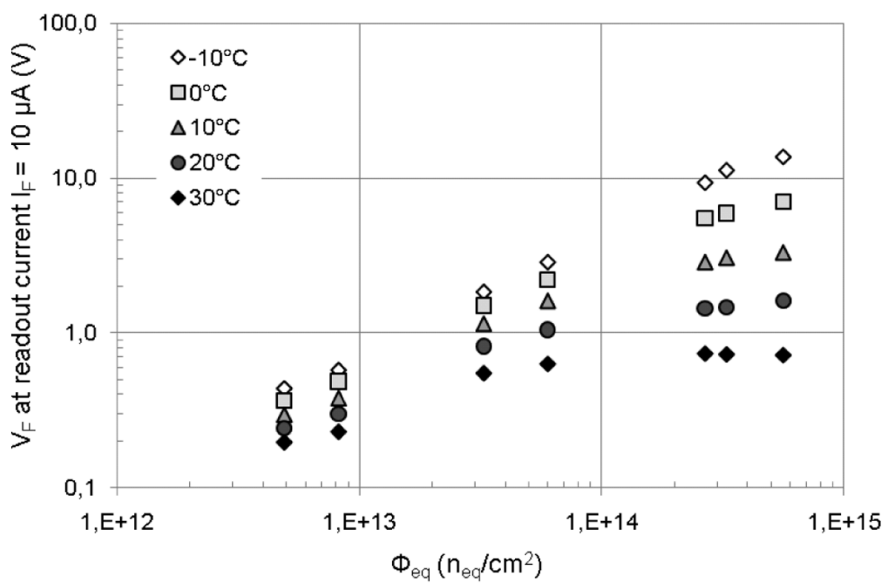

(a)

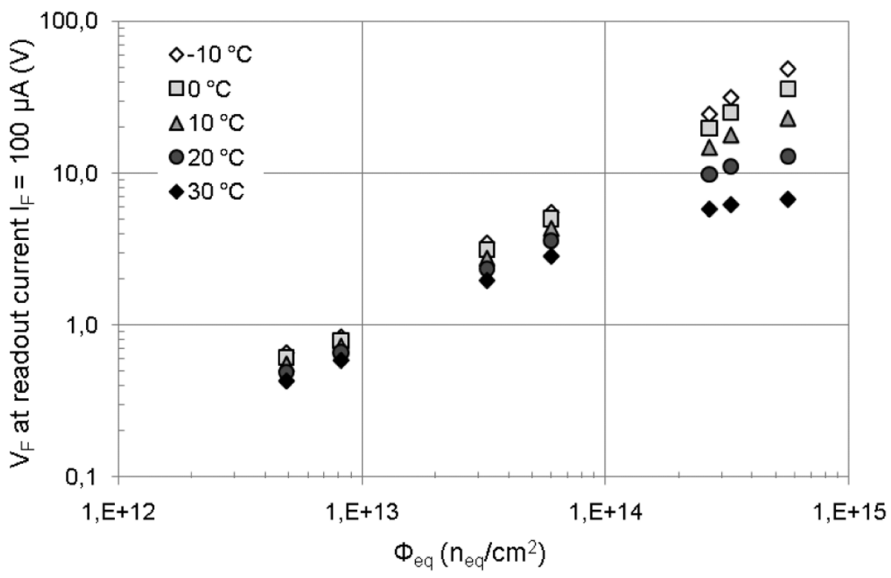

(b)

Fig. 8. Response of BPW34FS p-i-n diodes versus $\Phi_{\mathrm{eq}}$ at five different temperatures: $-10^{\circ} \mathrm{C}, 0^{\circ} \mathrm{C}, 10^{\circ} \mathrm{C}, 20^{\circ} \mathrm{C}$ and $30^{\circ} \mathrm{C}$. The forward voltage was measured at (a) $10 \mu \mathrm{A}$ and (b) $100 \mu \mathrm{A}$ readout current pulse of $50 \mathrm{~ms}$ pulse duration.

slightly rises up to higher fluences before saturating. It means that the maximum silicon resistivity is higher at lower temperature.

The value of $\rho_{\max }$ changes with temperature since the intrinsic carrier concentration $n_{i}$ is temperature dependent [27].

Therefore, at lower temperature, the maximum resistance of the BPW34FS will increase leading to an expanded upper-limit fluence range for the application of the diode as radiation monitor.

The same remarks can be made for Fig. 7 anf Fig. 8(b) when BPW34FS is operated at $100 \mu \mathrm{A}$.

The major difference is that, at this readout current, the fluence at which the diode becomes not sensitive to radiation damage anymore $\left(V_{F}\right.$ saturation) is higher than the ones observed at $I_{F}=10 \mu \mathrm{A}$.

This means that the "ohmic-like" behavior extends to $I_{F}=$ $100 \mu \mathrm{A}$ at higher fluences (see discussion in Section III) and so $V_{F}$ saturates at higher fluences (when the diode is ohmic-like).

In addition, it should be mentioned that an investigation of the temperature dependence on complete forward and reverse $I-V$ characteristics, from $-40^{\circ} \mathrm{C}$ to $20^{\circ} \mathrm{C}$ is still in progress and will be addressed in a future paper. 


\section{CONCLUSION}

In this paper, effects of radiation damage up to $6.3 \times 10^{15} \mathrm{n}_{\mathrm{eq}} / \mathrm{cm}^{2}$ on the OSRAM BPW34FS commercial $\mathrm{p}$-i-n diodes response have been studied.

BPW34FS silicon p-i-n diodes are already used as radiation sensors in LHC experiments, where detectors are exposed to radiation level up to a few $10^{14} \mathrm{n}_{\mathrm{eq}} / \mathrm{cm}^{2}$. In view of using them for monitoring up to 10 times higher fluences in future (Super-LHC) applications, a formulation to parameterize the radiation response of this device up to very high fluences has been presented and applied to experimental data obtained at room temperature after irradiation with $24 \mathrm{GeV} / \mathrm{c}$ protons up to $6.3 \times 10^{15} \mathrm{n}_{\mathrm{eq}} / \mathrm{cm}^{2}$.

The parameterization of the forward voltage as function of fluence and readout current is obtained by fitting a series of forward $I-V$ characteristics of diodes exposed to different fluences in the fluence range of interest with a two parameters function. The parameterization of the experimental data obtained in this work at room temperature was found to be valid for $\Phi_{\mathrm{eq}} \geq$ $10^{13} \mathrm{n}_{\mathrm{eq}} / \mathrm{cm}^{2}$ and a forward readout current of $I_{F} \leq 1 \mathrm{~mA}$. It can therefore be used to replace the previous readout protocol, which was limited to a fixed readout current value of $1 \mathrm{~mA}$ only. The flexibility to use an arbitrary readout current below or equal to $1 \mathrm{~mA}$ will allow to extend the fluence range of the BPW34FS diodes up to some $10^{15} \mathrm{n}_{\mathrm{eq}} / \mathrm{cm}^{2}$.

In addition, the temperature dependence of the voltage at constant readout current was qualitatively evaluated. It revealed that the upper limit of the fluence measurement range can be expanded by measuring the $\mathrm{p}-\mathrm{i}-\mathrm{n}$ diodes at lower temperature. A more comprehensive investigation on the temperature dependence of $I-V$ characteristics with the aim to integrate the temperature as a parameter into the parameterization of the diode voltage response is under way and will be the subject of a later paper.

\section{REFERENCES}

[1] The Large Hadron Collider Technical Design Report, CERN-2004003, 2004, 1-4.

[2] F. Ruggiero, "LHC Luminosity and Energy Upgrade: A Feasibility Study," CERN, LHC Project Report 626, Dec. 2002 [Online]. Available: http://cern.ch/lhc-proj-IR-upgrade/

[3] F. Gianotti, "Physics Potential and Experimental Challenges of the LHC Luminosity Upgrade," CERN, 2002, hep-ph/0204087.

[4] BPW34 Silicon p-i-n Photodiode Datasheet, OSRAM Opto-Semiconductors [Online]. Available: http://www.osram-os.com

[5] L. Malfante, "Utilisation de Dosimètres Semi-Conducteurs au Silicium Autour des Accélérateurs de Particules à Haute Energies," CERN-TISCFM/IR/92-09, 1992.

[6] F. Ravotti, M. Glaser, M. Moll, K. Idri, J. R. Vaillé, H. Prevost, and L. Dusseau, "Conception of an integrated sensor for the radiation monitoring of the CMS experiment at the large hadron collider," IEEE Trans. Nucl. Sci, vol. 51, pp. 3642-3648, 2004.

[7] F. Ravotti, M. Glaser, M. Moll, and F. Saigné, "BPW34 commercial p-i-n diodes for high level $1-\mathrm{MeV}$ neutron equivalent fluence monitoring," IEEE Trans. Nucl. Sci, vol. 55, pp. 2133-2140, 2008.

[8] F. Ravotti, "Development and characterization of radiation monitoring sensors for the high energy physics experiments of the CERN LHC accelerator," Ph.D. thesis, Dept. Electronics, Univ. Montpellier II, Montpellier, France, 2006, Also in CERN Thesis collection CERN-THESIS2007-013.

[9] M. McPherson, B. K. Jones, and T. Sloan, "Suppression of irradiation effects in gold-doped silicon detectors," J. Phys., vol. D 30, pp. 3028-3035, 1997.
[10] M. McPherson, B. K. Jones, and T. Sloan, "Effect or radiation damage in silicon p-i-n photodiodes," Semicond. Sci. Technol., vol. 12, pp. 1187-1194, 1997.

[11] B. K. Jones, J. Santana, and M. McPherson, "Semiconductor detectors for use in high radiation damage environments-Semi-insulating GaAs or silicon?," Nucl. Inst. Meth., vol. A 395, pp. 81-87, 1997.

[12] J. Santana and B. K. Jones, "Semi-insulating GaAs as a relaxation semiconductor," J. Appl. Phys., vol. 83, no. 12, pp. 7699-7705, 1998.

[13] B. K. Jones and M. McPherson, "Radiation damaged silicon as a semiinsulating relaxation semiconductor: Static electrical properties," Semicond. Sci. Technol., vol. 14, pp. 667-678, 1999.

[14] M. McPherson, "Fermi level pinning in irradiated silicon considered as a relaxation-like semiconductor," Nucl. Inst. Meth., vol. B 344, pp. $52-57,2003$.

[15] M. McPherson, "The space charge relaxation behavior of silicon diodes irradiated with $1 \mathrm{MeV}$ neutrons," Nucl. Inst. and Meth., vol. A 517, pp. 42-53, 2004.

[16] L. Dehimi, N. Sengouga, and B. K. Jones, "Modelling of semi-conductor diodes made of high defect concentration, irradiated, high resistivity and semi-insulating material: The current-voltage characteristics," Nucl. Inst. Meth., vol. A 519, pp. 532-544, 2004.

[17] A. B. Rosenfeld et al., "P-I-N diodes with a wide measurement range of fast neutron doses," Rad. Prot. Dos., vol. 33, no. 1/4, pp. 175-178, 1990.

[18] A. B. Rosenfeld et al., "Neutron dosimetry, with planar silicon p-i-n diodes," IEEE Trans. Nucl. Sci, vol. 50, pp. 2367-2372, 2003.

[19] I. Anokhin, O. Zinets, A. Rosenfeld, M. Lerch, M. Yudelev, V. Perevertaylo, M. Reinhard, and M. Petasecca, "Studies of the characteristics of a silicon neutron sensors," IEEE Trans. Nucl. Sci, vol. 56, pp. 2290-2293, 2009.

[20] M. Glaser, F. Ravotti, and M. Moll, "Dosimetry assessments in the irradiation facilities at the CERN-PS accelerator," IEEE Trans. Nucl. Sci, vol. 53, pp. 2016-2022, 2008.

[21] J. Mekki, "Characterization and performance optimization of radiation monitoring sensors for high energy physics experiments at the CERN LHC and Super-LHC," Ph.D. thesis, Dept. Electronics, Univ. Montpellier II, Montpellier, France, 2009.

[22] M. Moll, E. Fretwurst, M. Kuhnke, and G. Lindström, "Relation between microscopic defects and macroscopic changes in silicon detector properties after hadron irradiation," Nucl. Inst. Meth., vol. B186, pp. 00-110, 2002.

[23] W. V. Roosbroeck and H. C. Cassey, "Transport in relaxation semiconductors," Physical. Review B., vol. 5, no. 6, pp. 2154-2155, 1972.

[24] H. J. Queisser, "Semiconductors in the relaxation regime," in Proc. Eur. Solid-State Device Research Conf. (ESSDERC'72), Lancaster, U.K., Sep. 12-15, 1973, pp. 145-188, ISSN 0305-4632.

[25] N. M. Haegel, "Relaxation semiconductors: In theory and in practice," J. Appl. Phys., vol. A53, pp. 1-7, 1991.

[26] B. Anderson and R. Anderson, Fundamentals of Semiconductors Devices, 1st ed. New York: McGraw-Hill Science/Engineering/Math, 2004.

[27] S. M. Sze, Semiconductor Devices—Physics and Technology, 2nd ed. New York: Wiley, 2001.

[28] P. J. Walsh, R. Vogel, and E. J. Evans, "Conduction and electrical switching in amorphous chalcogenide semiconductor films," Phys. Rev., vol. 178, no. 3, pp. 1274-1279, 1969.

[29] T. J. Brodbeck, A. Chilingarov, T. Sloan, E. Fretwurst, M. Kuhnke, and G. Lindstroem, "Carrier mobilities in irradiated silicon," Nucl. Inst. Meth., vol. A 477, pp. 287-292, 2002.

[30] LHC Experiment Radiation Monitoring Working Group (RADMON) [Online]. Available: http://hc-expt-radmon.web.cern.ch/lhc-exptradmon

[31] F. Ravotti et al., Sensor Catalogue-Data Compilation of Solid-State Sensors for Radiation Monitoring CERN TS-Note-2005-002, 2005.

[32] R. M. Corless, G. H. Gonnet, D. E. G. Hare, D. J. Jeffrey, and D. E. Knuth, "On the LambertW function," Advances in Computational Mathematics, vol. 5, pp. 329-35.

[33] T. C. Bandwell and A. Jayakumar, "Exact analytical solution for current flow through diode series resistance," Electronics Lett., vol. 36, no. 4, pp. 291-292, 2000.

[34] P. Hruska, Z. Chobola, and L. Grmela, "Diode I-U curve fitting with Lambert W function," presented at the Int. Conf. Microelectronics (MIEL 2006), Belgrade, Serbia and Montenegro, May 14-17, 2006.

[35] W. Jung and M. Guziewicz, "Schottky diode parameters extraction using Lambert W function," Mater. Sci. Eng. B, 2009, doi: 0.1016/j. mseb.2009.02.013. 